\title{
Assessment Method of Facial Palsy by Amount of Feature Point Movements at Facial Expressions
}

\author{
Toshiyuki Tanaka Member (Keio University, tanaka@appi.keio.ac.jp) \\ Junko Nemoto Non-member, Manami Ohta Non-member \\ Takanobu Kunihiro Non-member
}

Keywords: facial palsy, feature point movement, assessment system, facial expression

At present, in the medical field the 40 point method and facial nerve grading system are generally used for the assessment of facial palsy. They are the methods that doctors express numerically the difference of right and left side on the face, and evaluate the palsy severity. However, there are some problems in terms of objectivity and reproducibility, and these methods have the limitation in the precise assessment, because clinic result is influenced by subjectivity of each doctor. It is also difficult to understand the condition of the patients' recovery with those assessment methods. Although various attempts have been performed to solve these problems, they have not been widely applied to the real diagnosis either.

The purpose of this paper is to make some indices based on the motion vector of the reference points on the face int the facial expression motion, and to apply the indices to a quantitative assessment of facial palsy. In this paper, we note characteristics of the expression muscles to decide the feature points on the face. We numerically express the movement of the feature points arranged along the expression muscles. We use color information for deciding facial position in the image. More precise contour extraction is performed using region extraction method with uniform $\mathrm{HVC}$ color space. In many examples, a facial nerve symptom appears in either of left and right on the face. Therefore, we propose the indices based on the symmetry of the facial motion. The following five facial expressions (including the rest) are used in this paper; Rest, Grin, Whistle, Eye closure and Wrinkling up forehead. Moreover, we make total assessment by adding synkinesis to the indices.

We compute our proposed indices for 31 palsy subjects

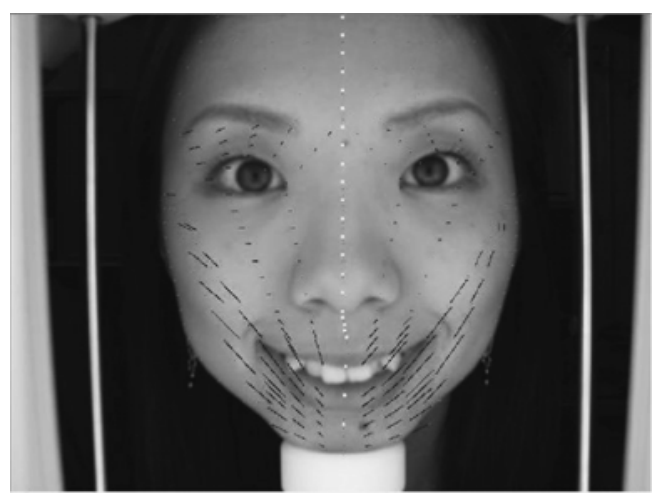

Fig. 1. Face fixed equipment for experiment and 5 healthy persons. Figure 1 shows the motion vector obtained with our method. Since we used four facial expressions among those of the 40 point method, the maximum score of both method is 16 . Our method has the positive correlation to the score of the 40 point method as seen in Fig.2. Although the 40 point method is scored with discrete point by 0,2 and 4, our method is scored with analog point. Comparison of our method and the 40 point method shows that range of score 2 by the 40 point method is wider than those of score 0 and 4 as said from the past. Although one does not show the results for two facial motions (eye closure and wrinkling up forehead), those are similar to the obtained result. It seems from a total estimation that the score obtained by our method is a little greater than that of the 40 point method. The reason seems that score of our method has some small value even if score of the 40 point method is 0 . We compared our proposed indices of each facial expression with those of the 40 point method. As result of observing the process of patient recovery, we could represent detail of recovery process which was difficult in the 40 point method. In addition, when we integrated each index as total assessment, we found the correlation of our method with the 40 point method at an extent. We achieved more comprehensive assessment for facial palsy, since we could use more facial expressions than the previous method using contour extraction of facial feature. It seems from the obtained results that our proposed motion vector method is applied to the quantitative assessment of facial palsy, and that it is effective as a diagnosis support system.

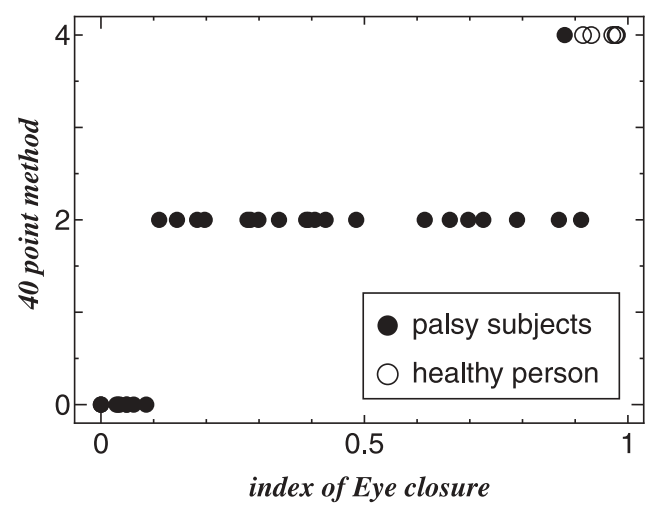

Fig. 2. Comparison of our proposed method with the 40 point method in facial expression (Eye closure) 


\title{
Assessment Method of Facial Palsy by Amount of Feature Point Movements at Facial Expressions
}

\author{
Toshiyuki Tanaka* Member \\ Junko Nemoto* Non-member \\ Manami Ohta* Non-member \\ Takanobu Kunihiro** $^{*}$ Non-member
}

\begin{abstract}
At present in medical field, the 40 point method and facial nerve grading system (House-Brackmann method) are generally used for assessment of facial palsy. However, those methods have limitation in the precise assessment, because of subjectivity in diagnosis. Purpose of this paper is to propose objective and quantitative assessment of facial palsy based on the amount of feature point movements on the face. Facial nerve symptoms generally appear in either of right and left side on the face. In facial expression of palsy subjects, the motion on the diseased side becomes smaller than that on the healthy side. We defined some indices of palsy severity from the observation of facial expression. Those indices showed the asymmetry of the facial motion quantitatively. We confirmed that our proposed method was valid for assessment of the facial palsy by comparison with the 40 point method.
\end{abstract}

Keywords: Facial palsy, feature point movement, assessment system, facial expression

\section{Introduction}

At present in Japan, the 40 point method are generally used for the assessment of facial palsy in the medical field. A facial nerve grading system (House-Brackmann method) ${ }^{(1)}$ is also used in foreign countries. They are the methods that doctors score the expressions of palsy subject based on the difference of right and left facial motion when the palsy subject performs the 10 facial expression test. The doctors assess the palsy severity from the score. However, there are the problems that the scores of each doctor are different, and that the score does not have sufficient reproducibility. Moreover since those methods have the limitation in the precise assessment, it is difficult for doctors to understand the recovery of the patients. Although various attempts have been performed to solve those problems ${ }^{(2)-(5)}$, they have not been widely applied to the real diagnosis either.

The following researches are reported as computeraided objective diagnosis method of facial palsy for compensating the 40 point method : A method with optical flow ${ }^{(3)}$, a method with shape extraction ${ }^{(4)}{ }^{(5)}$, a method with marker ${ }^{(6)}$, a method with moiré stripe ${ }^{(7)}$ and so on. Although those approaches are familiar in the field of engineering such as image processing, it is difficult to make the valid indices for facial palsy from the obtained results. One reason of the difficulties is that there is

\footnotetext{
* Department of Applied Physics and Physico-Informatics, Faculty of Science and Technology, Keio University,

3-14-1 Hiyoshi, Kohoku-ku, Yokohama 223-8522

** Department of Otorhinolaryngology, School of Medicine, Keio University,

35 Shinanomachi, Shinjuku-ku, Tokyo 160-8582
}

not reference data for studies except the scores of the 40 point method, and another reason is the difficulty of searching the corresponding points of the both side on the face for computing the indices. Since symptoms of facial palsy mainly appear on either side of right and left on the face, most of researches for facial palsy use the movable difference of right and left side on the face. Therefore it is important to decide the corresponding points on both side of the face.

The purpose of this paper is to make some indices based on the motion vectors of the reference points on the face in the facial expression, and to apply the indices to a quantitative assessment of facial palsy. First, we obtain a facial position in the image as a rectangle area, and a facial contour is obtained within the rectangle. Besides 4 feature points of eyes anf mouth are given by clicking a computer mouse. We automatically decide other feature points for assessing a facial nerve from the facial contour and the given feature points, considering characteristics of some expression muscle. We make some indices based on the symmetry of 5 facial motions : Rest, Grin, Whistle, Eye closure, and Wrinkling forehead. We compare scores of our proposed method with those of the 40 point method.

\section{Methodology}

We here explain an emvironment of experiment, a decision of facial position, an extraction of facial contour, a region division by facial muscle, and indices for diagnosis of facial palsy.

\subsection{Environment of Experiment}

Experiments of our system are performed at Keio Uni- 


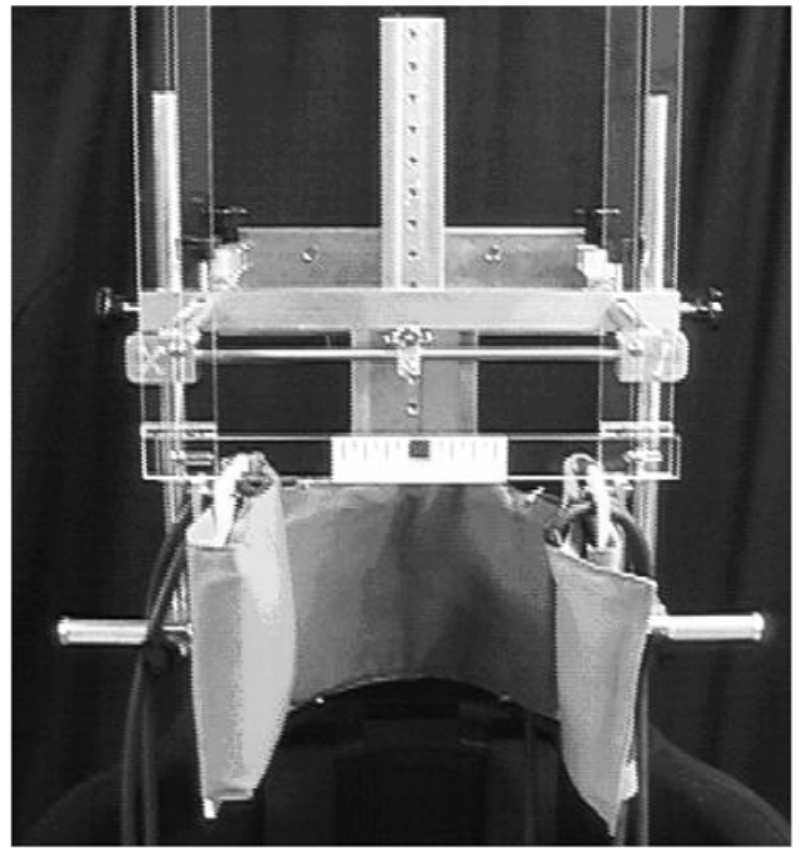

Fig. 1. Face fixed equipment for experiment

versity Hospital. All the images for our research are taken with the video camera SONY DCR-TRV10. The images are sent to a computer though the video-capture board (Canopus DVRapter). Those images are saved as digital images with $640 \times 480$ pixel and 24 bit color data. When the photographing is performed, a face of subject is fixed by an equipment which is shown in Fig.1. The video camera is set at the distance of $1.5 \mathrm{~m}$ from the subject. Each subject performs 5 sorts of facial expressions shown in the following.

(1) Rest

(2) Grin

(3) Whistle

(4) Eye closure weakly

(5) Wrinkling up forehead

\subsection{Decision of Facial Position}

First, we decide a facial position in the digital images that are taken with video camera. Since it is known that I component of YIQ color basis ${ }^{(8)(9)}$ corresponds to a facial color of Japanese, we use the I component of digital images. In YIQ color basis, Y component means the luminance, which is just the brightness of monochrome images, I component is essentially red minus cyan, and $\mathrm{Q}$ component is magenta minus green. Transformation from RGB basis to YIQ basis is shown below.

$$
\left[\begin{array}{l}
Y \\
I \\
Q
\end{array}\right]=\left[\begin{array}{ccc}
0.299 & 0.587 & 0.114 \\
0.596 & -0.274 & -0.322 \\
0.211 & -0.522 & 0.311
\end{array}\right]\left[\begin{array}{l}
R \\
G \\
B
\end{array}\right]
$$

We make an image brightness histogram from I component images, and perform linear conversion for the histogram using the following equation.

$$
y_{j}=\frac{y_{D}-y_{C}}{x_{B}-x_{A}}\left(x_{i}-x_{A}\right)+y_{C} \ldots \ldots \ldots \ldots \ldots
$$

In the above equation intensity range $\left[x_{A}, x_{B}\right]$ is converted into the range $\left[y_{C}, y_{D}\right]$. Binarization for the digital image is performed by a discriminant method ${ }^{(10)}$ for the obtained histogram. An expansion processing and a shrinking processing are performed after binarization. We obtain a facial shape after shrinking, expanding, and labeling processing. The facial position in the image is obtained as a rectangle area that is sufficient size for including the whole shape of the face.

\subsection{Extraction of Facial Contour}

Next, we decide a facial contour within the obtained rectangle region. The contour extraction of face includes 4 steps: conversion of RGB color basis to HVC color basis as step 1, filtering of heu $(\mathrm{H})$, brightness $(\mathrm{V})$ and saturation (C) with median filter, and emphasis for $\mathrm{H}$ and $\mathrm{C}$ image using human visual nature as step 2, decision of facial shape using color difference equation as step 3, transformation of HVC image to YIQ image and transformation of YIQ image to RGB image for displaying as step 4.

RGB color basis is converted into HVC basis in the following process. RGB images are converted to CIE(1934)XYZ basis.

$$
\begin{aligned}
& {\left[\begin{array}{l}
X \\
Y \\
Z
\end{array}\right]=\left[\begin{array}{lll}
0.608 & 0.174 & 0.200 \\
0.299 & 0.587 & 0.144 \\
0.000 & 0.066 & 1.112
\end{array}\right]\left[\begin{array}{l}
R \\
G \\
B
\end{array}\right]} \\
& \text {................. (3) } \\
& x=X /(X+Y+Z) \cdots \cdots \cdots \cdots \cdots \cdots \cdots \\
& y=Y /(X+Y+Z)
\end{aligned}
$$

HVC color basis is obtained with transformation table ${ }^{(8)}$ from $(x, y, Y)$ data in the above equation. However, since this table do not have enough data for transformation, we convert the obtained $(x, y, Y)$ data into HVC image using Mathematical Transform to Munsell (MTM). MTM method is omitted in detail, since the explanation of MTM requires some pages. MTM method is reported in some reference ${ }^{(11)}$.

In the step 2, the filtering is performed with median filter for brightness (V) and saturation (C) components of the obtained images. We use the filter with size of $3 \times 3$. Next, $\mathrm{V}$ and $\mathrm{C}$ components in HVC image are emphasized by the method that models after human visual nature. As the modeling nature, man performs the compensation by the relative brightness, compensation by the differential brightness, compensation by the brightness conversion, and compensation by relative saturation.

(i) Compensation by the relative brightness

This compensation emphasizes the brightness of region that has low contrast, and it is used in order to restrain brightness of region that brightness value suddenly changes like highlight. Relative brightness $V_{r}(X)$ is expressed below when reference pixel is $X$, brightness of reference pixel is $V_{c}(X)$ and average brightness of peripheral parts of reference pixel is $V_{s}(X)$.

$$
V_{r}(X)=f_{v}\left(\frac{V_{c}(X)}{V_{s}(X)}\right)
$$




$$
f_{v}(x)= \begin{cases}0 & x<0 \\ x & 0 \leq x<1 \quad \ldots \ldots \ldots \\ \log (x)+1 & 1 \leq x\end{cases}
$$

(ii) Compensation by the differential brightness

This compensation is carried out to emphasize the light and dark boundary line. Differential brightness $V_{d}(X)$ of reference pixel $X$ is expressed as the following equation.

$$
V_{d}(X)=V_{c}(X)-V_{s}(X)
$$

(iii) Compensation by the brightness conversion

Contrast of whole image is low, when brightness is computed by relative brightness and differential brightness, because it has only local brightness information in the image. Therefore, it is better that brightness is computed by adding brightness of original image. Three elements are linearly combined with weights for the elements as shown in the following equation. Brightness $V(X)$ that is normalized from the obtained brightness is regarded as consequent brightness. The former brightness in reference pixel $X$ is $V_{o}(X)$. The weighting factor of relative brightness is $d_{v 1}$. The ratio of the former brightness to relative brightness is $k_{v 1}$. The weighting factor of differential brightness is $k_{v 2}$.

$$
\begin{array}{r}
V(X)=d_{v 1} V_{r}(X)+\left(1-k_{v 1}\right) V_{o}(X)+k_{v 2} V_{d}(X) \\
\ldots \ldots \ldots \ldots \ldots \ldots \ldots
\end{array}
$$

(iv) Compensation by relative saturation

Technique similar to relative brightness is also applied in relative saturation. For both high saturation region and low saturation region, tone difference is expanded in local region. Converted saturation $S(X)$ is expressed by the following equation, when saturation of reference pixel is $S_{c}(X)$, average saturation of local region on reference pixel $X$ is $S_{s}(X)$, saturation of reference pixel in original image is $S_{o}(X)$, relative saturation is $S_{r}(X)$ and ratio of relative saturation to saturation of original image is $k_{s 2}$.

$$
\begin{aligned}
& S_{r}(X)=f_{v}\left(\frac{S(X)}{S_{s}(X)}\right) \\
& f_{v}(x)= \begin{cases}0 & x<0 \\
x & 0 \leq x<1 \\
\log (x)+1 & 1 \leq x\end{cases} \\
& S(X)=k_{s 2} S_{r}(X)+\left(1-k_{s 2}\right) S_{o}(X)
\end{aligned}
$$

After those compensations, we decide a precise facial shape using color difference equation in step 3 . The color difference means the color of perception.

NBS color difference and Godlove color difference are known as the representative color difference. When we compare NBS color difference with Godlove color difference for our method, the result with Godlove color difference has better performance than NBS color difference. We therefore selected Godlove difference equation. Color difference value $\mathrm{E}$ (Godlove) is expressed as below when HVC values for 2 colors are $\left(H_{1}, V_{1}, C_{1}\right)$ and $\left(H_{2}, V_{2}, C_{2}\right)$, respectively.

$$
\begin{aligned}
& \Delta E(\text { Godlove })=\left(2 C_{1} C_{2}(1-\cos (2 \pi \Delta H / 100))\right. \\
& \left.+\Delta C^{2}+(4 \Delta V)^{2}\right)^{1 / 2} \text {. } \\
& \Delta H=\left|H_{1}-H_{2}\right| \\
& \Delta V=\left|V_{1}-V_{2}\right| \\
& \Delta C=\left|C_{1}-C_{2}\right|
\end{aligned}
$$

The precise facial shape is extracted by the nature that the color difference equation has big value near edge of the object.

Step 4 is the procedure for the display of the obtained image. HVC image is converted into YIQ image using the inverse transformation of MTM method. YIQ image is converted into RGB image using the inverse transform of equation (1).

\subsection{Decision of Reference Points by Facial Muscle}

After the extraction of facial shape in the previous process, we arrange the reference points within the facial shape. Since our system is not perfectly automatic, several reference points must be manually marked within the facial region. The following points are marked by clicking the computer mouse.

(i) For eyes : inner corner of eyes, outer corner of eyes, center of top eyelid, center of bottom eyelid

(ii) For mouth : right and left corner of mouth, center of upper lip, center of lower lip

(iii) For nose : center beneath nose

(iv) For eyebrow : inner corner of eyebrow, top of eyebrow, outer corner of eyebrow

From the obtained facial shape by the method in previous section, we automatically obtain the reference points on the facial contour by dividing the shape into 24 small regions sliced vertically and equally. Next, center line of face is obtained as piecewise linear lines. The lines is selected that goes through center of inner corner of eyes and center beneath nose from forehead to nose, that goes through center beneath nose and center of upper lip from nose to upper lip, and that goes through center of lower lip and center of chin.

The facial muscle ${ }^{(12)-(17)}$ is necessary to move the eyes, the mouth, the nose, and the ears and so on. We think that the following seven muscle are important for our assessment method of facial palsy.

(1) venter frontalis

(2) m. orbicularis oculi

(3) m. corrugator supercilii

(4) m. zygomaticus mafor

(5) $\mathrm{m}$. risorius

(6) $\mathrm{m}$. depressor anguli oris

(7) m. depressor labii inferioris

From the given reference points, the automatically computed reference points, and the computed center of face, we divide the facial shape into 8 small regions in each side in consideration of the arrangement of facial muscles as shown in the black lines of Fig.2. Except the black lines, we obtain several reference lines in consider of the above facial muscle, as shown in Fig.2. We divide the obtained lines into 24 segment in the vertical direction and 6 segments in the right and left direction. 


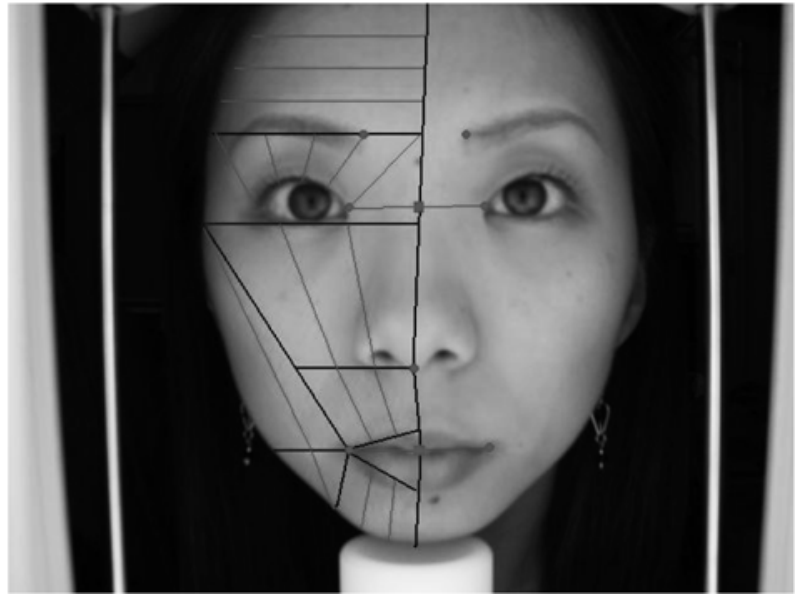

Fig. 2. Small partitions within the face for obtaining the reference points

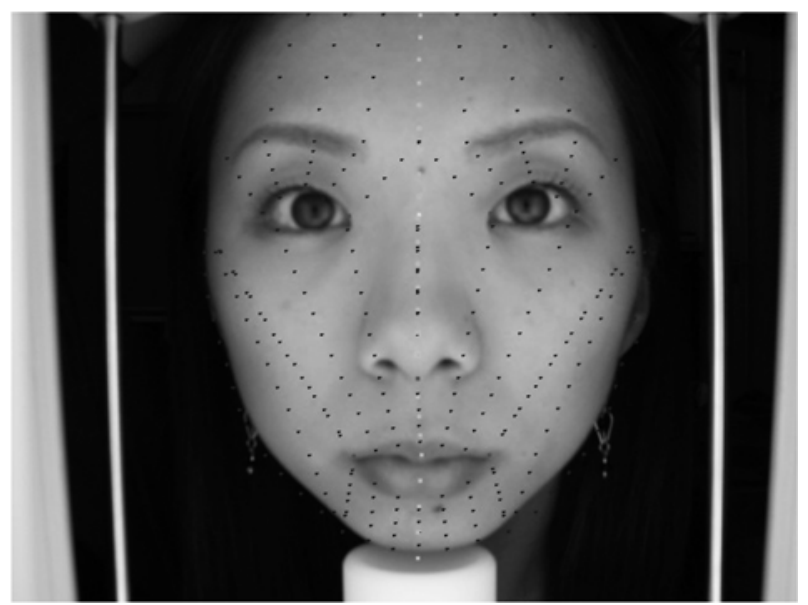

Fig. 3. Reference points obtaining the motion vectors in facial expression

The reference points of our method are obtained in the nodes of the vertical and horizontal division, and are 288 points in total, as shown in Fig.3. In the following section we use the reference points for performing diagnosis for facial palsy.

\subsection{Indices for Diagnosis of Facial Palsy}

We make some indices for 5 regions (A)-(E) as shown in Fig.4, except regions of both sides of nose and both upper and lower lips. Four facial expressions relate to some of the region $(\mathrm{A})-(\mathrm{E})$ as shown in Table 1 . We make indices from shift of reference points within the related regions shown in Table 1.

The indices do not have to be influenced by the shift of reference points except constriction of muscle. When the palsy becomes severer, the shift of the reference points becomes greater, because skin of healthy side pull skin of diseased side. For the indices of precise diagnosis, we implement the vector $v_{d}$ which means constriction of expression muscle in the diseased side, and the vector $v_{s}$ which means synkinesis pulling the skin. Those vectors are defined according to the direction of each vector.

(1) Grin: region $(\mathrm{C}),(\mathrm{D})$ and $(\mathrm{E})$

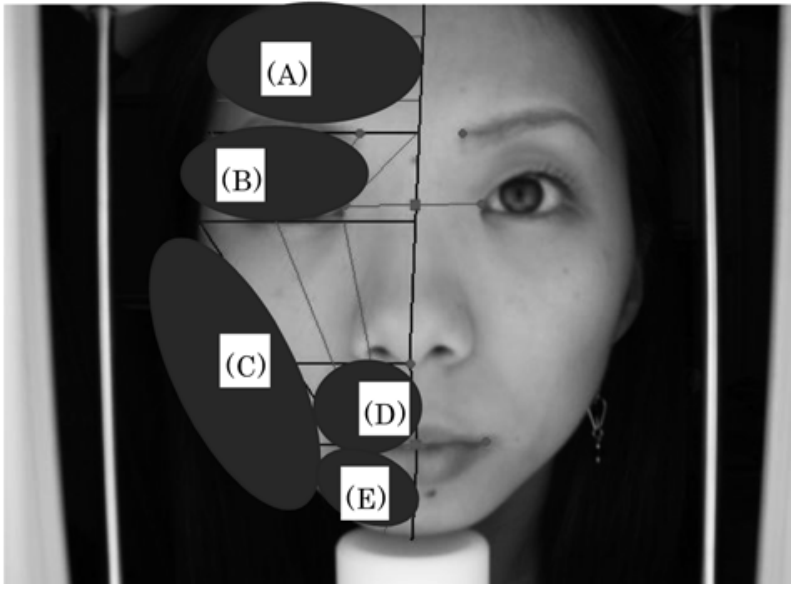

Fig. 4. Small regions within the face for making indices of facial palsy

Table 1. Corresponding region for each facial expression

\begin{tabular}{l|c}
\hline Facial Expressions & Regions \\
\hline Grin & $(\mathrm{C}),(\mathrm{D}),(\mathrm{E})$ \\
\hline Whistle & $(\mathrm{C}),(\mathrm{D}),(\mathrm{E})$ \\
\hline Eye closing weakly & $(\mathrm{B})$ \\
\hline Wrinkling up forehead & $(\mathrm{A})$ \\
\hline
\end{tabular}

We define an index $I_{G}$ for Grin motion.

$$
I_{G}=I-k_{s} I_{s}
$$

where $I$ means the index of normal facial expression and $I_{s}$ means the index of abnormal synkinesis. $k_{s}$ is the weighting factor of abnormal synkinesis. The solid line going through the reference points and its dashed perpendicular line are shown in the left image of Fig.5. We assume that the vector within light shaded region in Fig.5(a) is that of normal expression in cheek region (C), and that the vector out of shaded region is that of abnormal synkinesis. Since the lines through the reference points have the different slopes, and the shape of shaded region is decided by both lines, the shapes of shaded region are a little different from each other. We also assume that the shaded region in Fig.5(b) is normal direction of motion vectors in mouth region (D) and jaw region $(\mathrm{E})$.

$$
\begin{aligned}
& I=\sum_{X=C, D, E} k_{X} r_{X}=\sum_{X=C, D, E} k_{X} \frac{\sum_{X}\left|v_{d}\right|}{\sum_{X}\left|v_{o}\right|} \\
& \quad \ldots \ldots \ldots \ldots \ldots \ldots \ldots \ldots \ldots
\end{aligned}
$$

In the above equation, $\left|v_{o}\right|$ means the absolute value of vector on the healthy side, $\left|v_{d}\right|$ means the absolute value of vector on the diseased side, $\left|v_{s}\right|$ means the absolute value of vector by abnormal synkinesis, and $k_{X}$ means the weight coefficient.

(2) Whistle: region (C), (D) and (E)

An index for Whistle motion $I_{W}$ is similar to that of 


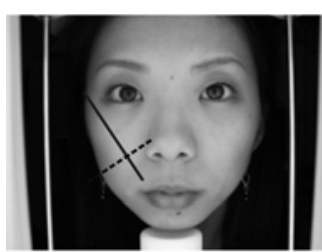

- parting line

-.-.-perpendicular

normal vector region

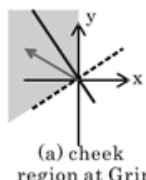

region at Grin

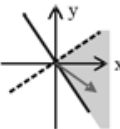

(c) cheek

egion at

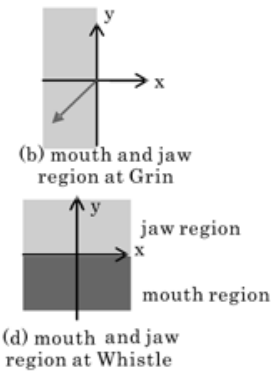

Fig. 5. Direction of normal vector of the healthy person in facial expression

Grin motion. The values of vectors are computed using eq.(17), (18) and (19) within the light shaded region of Fig.5(c),(d). We assume that the vectors within the light shaded region in Fig.5(c) and (d) are also those of normal expression, and the vectors in other region are ones of abnormal synkinesis.

(3) Eye closure: region (B)

An index $I_{E}$ for eye closure is defined as below.

$$
I_{E}=\frac{\sum_{E}\left|v_{d}\right|}{\sum_{E}\left|v_{o}\right|}-k_{d} \frac{d_{d}}{\operatorname{ave}\left(\left|v_{o}\right|\right)} \cdots \cdots \cdots \cdots \cdots
$$

In the above equation, $d_{d}$ means the distance between upper eyelid and lower eyelid on the diseased side. Eye closure is not enough precise index in our method when facial palsy is severe. In the 40 point method this index is important for diagnosis. Eqation (20) is modified by an average of $\left|v_{o}\right|$ for normalization of facial size.

(4) Wrinkling up forehead: region (A)

An index $I_{F}$ for wrinkling up forehead is defined as below. This index is a ratio of the vector on the diseased side and healthy side.

$$
I_{F}=\frac{\sum_{F}\left|v_{d}\right|}{\sum_{F}\left|v_{o}\right|} \ldots \ldots \ldots \ldots \ldots \ldots \ldots \ldots \ldots \ldots \ldots \ldots \ldots
$$

We make indices from the information of region (A)(E), and make the indices correspond to each facial motion. In the above description the indices of Grin and Whistle motion are computed using the vectors of abnormal synkinesis unlike other indices. Facial palsy includes complete paralysis that muscle cannot shrink at all, and palsy that muscle shrinks less than one of healthy side. We consider the vectors of abnormal synkinesis for reflecting the difference between complete palarysis and palsy.

\section{Result}

We computed our proposed indices for 31 palsy subjects and 5 healthy persons. Fig. 6 shows an example of a rectangular facial position in the image, which is obtained with I component of YIQ color basis. The facial contour obtained within the rectangular region is exacted as shown in the Fig.7. Fig.8 shows the motion vectors obtained with our method for a healthy person. Fig. 9 shows a result of comparison of our proposed method with the 40 point method when subjects performed Grin motion as facial expression. In the 40 point method the

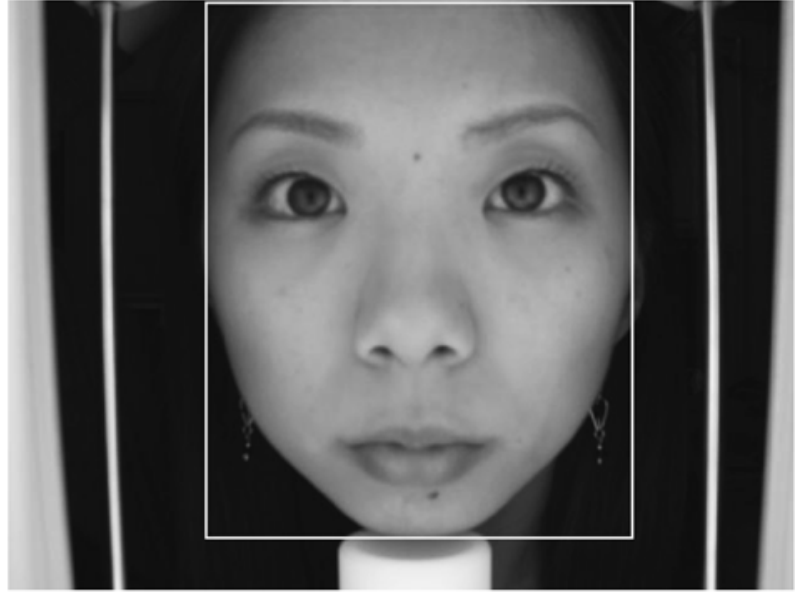

Fig. 6. Facial position is shown as a rectangular region, which is obtained with I component of YIQ color basis

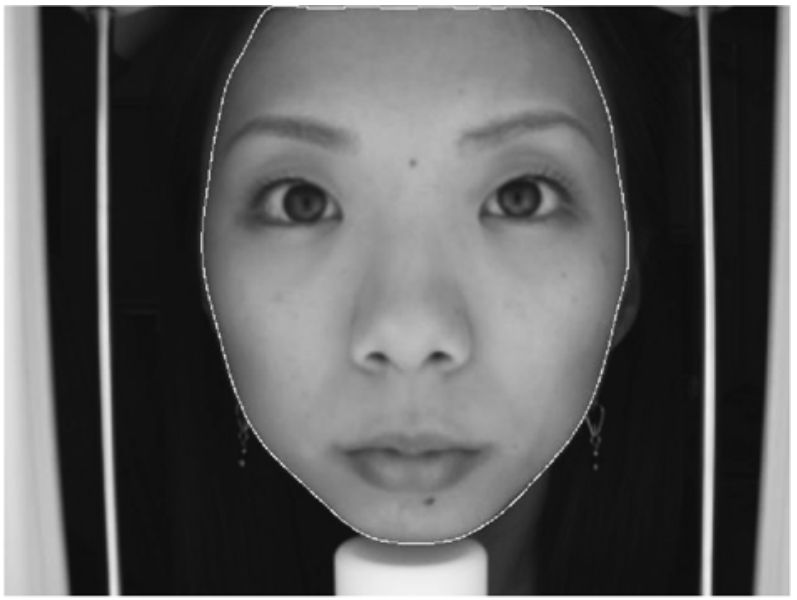

Fig. 7. Obtained facial contour using color difference equation

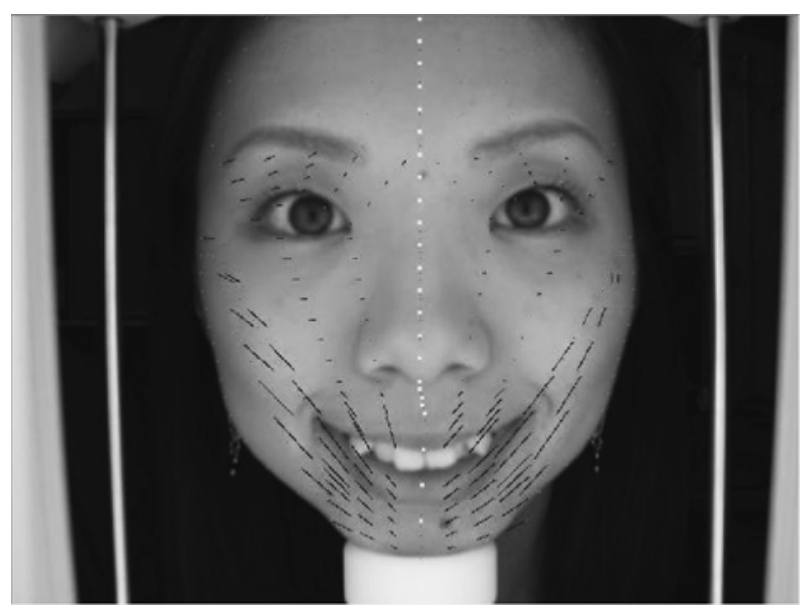

Fig. 8. Motion vectors in the facial expression (Grin)

discrete scores of 0,2 , and 4 are used, and those of our method are linear number that ranges from 0 to 1 . Grin motion seems to be the most effective expression among 10 facial expressions for facial palsy diagnosis. If the 40 


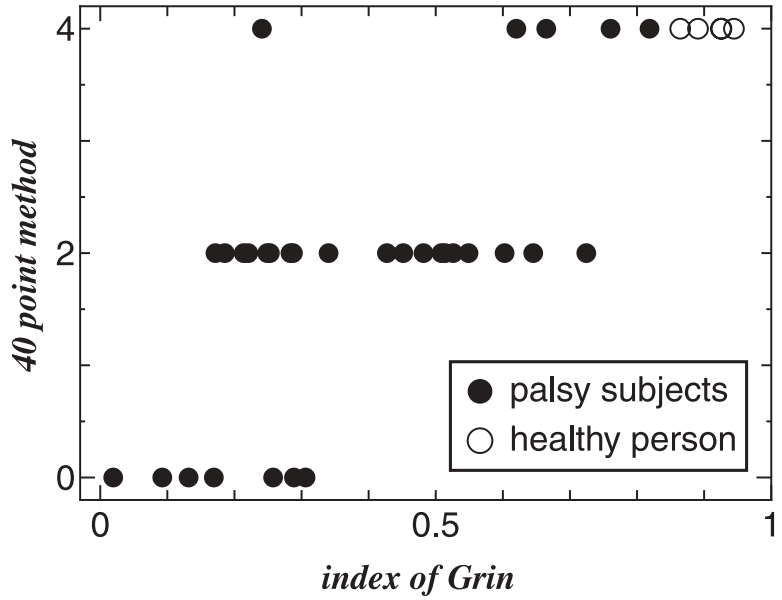

Fig. 9. Comparison of our proposed method with the 40 point method in facial expression (Grin)

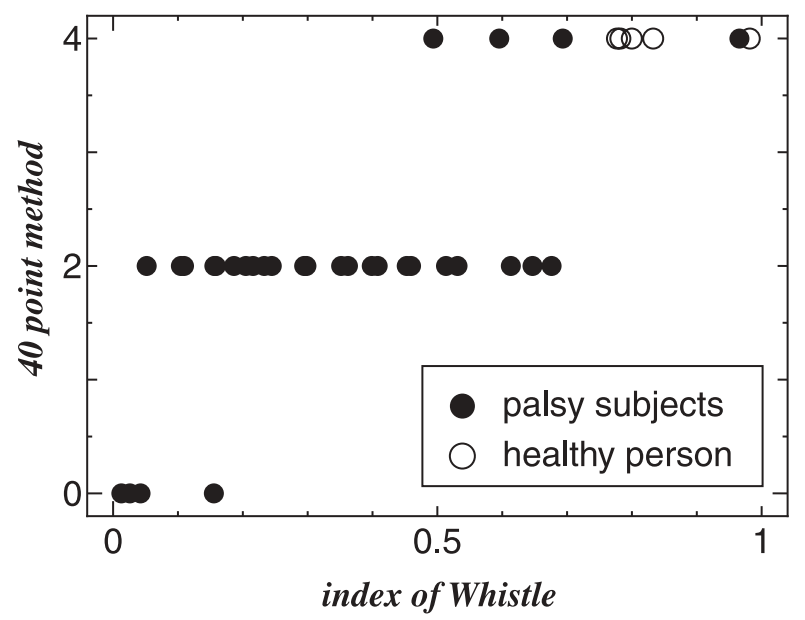

Fig. 10. Comparison of our proposed method with the 40 point method in facial expression (Whistle)

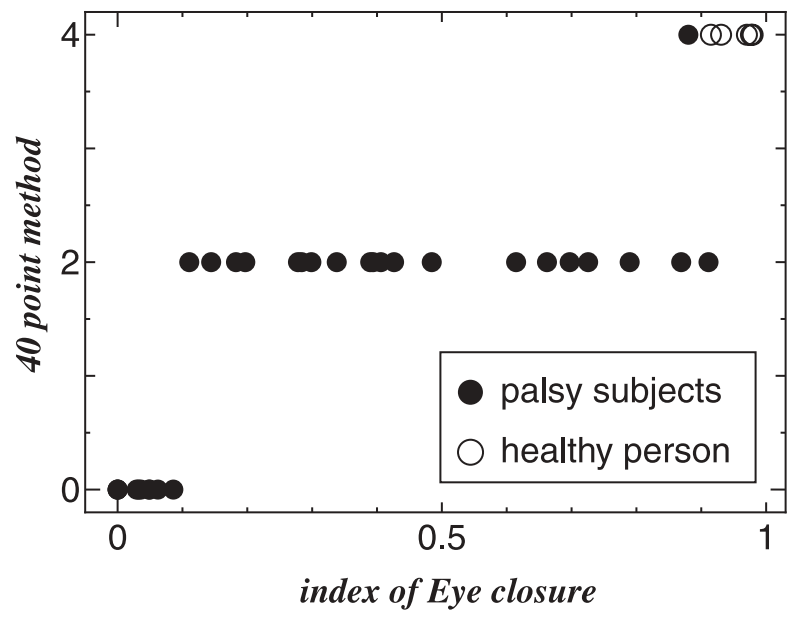

Fig. 11. Comparison of our proposed method with the 40 point method in facial expression (Eye closure)

point method and our proposed method perform exact diagnosis of facial palsy, Fig.9 becomes the graph with stepwise form. Although Fig.9 shows a relatively good

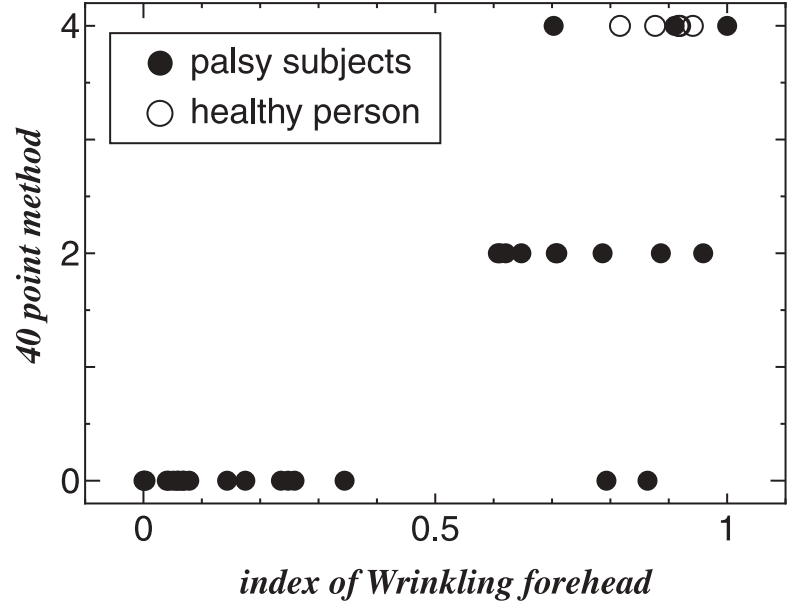

Fig. 12. Comparison of our proposed method with the 40 point method in facial expression (Wrinkling forehead)

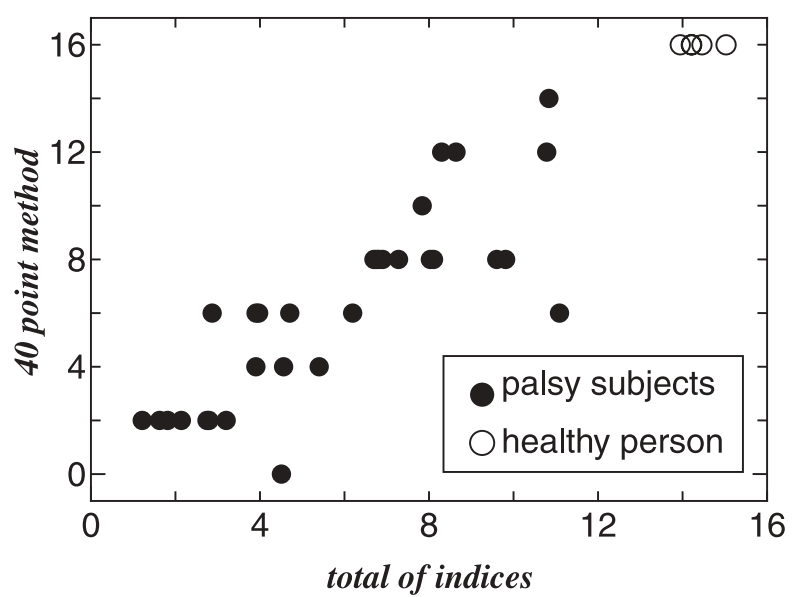

Fig. 13. Comparison of total score of our proposed method with the 40 point method

correlation, each score of the 40 point method spreads widely. It is especially close to the sense of doctor that score 2 has wide range, and that some different scores in the 40 point method are the same score in our method. When doctors score the facial expression, they often give score 2 if the motion is neither score 0 (complete paralysis) nor 4 (healthy). Besides the graph shows that palsy subjects with score 4 have smaller value than healthy persons with score 4 in our method. Fig.10 similarly shows a result of comparison when subjects performed Whistle motion as facial expression. The discrete number of 0,2 ,and 4 are used as the scores of the 40 point method, and the linear number that ranges from 0 to 1 are used as the score of our method. In this figure score 2 spreads to the range of smaller value. Score 0 and 2 overlap each other. We think that this results in the reason that doctor observes the whistle motion in three dimension, and on the other hand our method performs the diagnosis in two dimension. Fig.11 shows a result of comparison when subjects performed Eye closure motion. This figure shows that persons with score 
0 and 4 in the 40 point method have score nearly 0 and 1 in our method, respectively. Score 2 in the 40 point method spreads in the wide range. Fig. 12 shows a result of comparison when subjects performed Wrinkling forehead motion. Data with score 2 shift to greater value near 1.0 in our method. This reason seems that motion vectors become greater since the muscle in the healthy side pulls that in the diseased side. In the same reason some of the score 0 become greater value. Fig. 13 shows the comparison of total score of our method with that of the 40 point method. Since we used 4 facial expressions among those of the 40 point method except Grin, man computed the score of the 40 point method as the total score of 4 facial expressions. The score of our method is modified so that the maximum number will be 16 . Our method has the positive correlation to the score of 40 point method as seen in Fig.13.

We confirmed the results of our method were good credibility and robustness by repeating several times the computation of indices for the same images. The variance of indices for the same images was enough small to trust that our method give us the good reproducibility.

\section{Discussion}

Although an example of contour extraction of face is shown as Fig.7, other samples are similarly obtained exact facial contours by our proposed method. Motion vectors are computed as shown in Fig.8. In our method 2 images of the Rest and another facial expression are necessary for computation. Those vectors are precise and valid for indices for facial palsy. As shown in Figs.9, 10 , and 11 , data with score 2 in the 40 point method spread widely with respect to our score. In the 40 point method the score 0 means complete paralysis, the score 4 means healthy motion, and the score 2 means other cases. This scoring makes wide range of score 2 , and it is difficult for doctors to understand the recovery process of facial palsy. Since all the data with score 2 in the 40 point method correspond to different scores in our method, we think that our method performs more precise diagnosis than the 40 point method. While on the other hand, in the result of wrinkling forehead as shown in Fig.12, data with score 2 and 4 focus and overlap in the small range. The displacement in the forehead region is little difference between the motion of facial palsy and healthy person, except complete paralysis. In the result of whistle motion shown in Fig.10, data with score 4 spread to the region of small value. This reasons that although the vector of whistle motion is three dimensional, our method use the digital image in two dimension. It seems from a total assessment that the score obtained by our method is a little greater than that of the 40 point method. The reason seems that score of our method has some small value even if score of the 40 point method is 0 .

In this reseach our method is semi-automatic, and several reference points such as eyes and lips must be manually given. Although we tried to make automatic system, we could not obtain a sufficient performance of speed and accuracy. When we request more objectivity and reproducibility, manual input must be reduced. As further research, we wish to automatically extract the exact shape of eyes and lips using color information and other processing method. In this research we use 5 facial expression of 40 point method : Rest, Grin, Whistle, Eye closure, and Wrinkling forehead. Other 5 expressions require three dimensional information for making the indices. We think that our proposed method is better than previous objective method, but we require all of 10 expression of 40 point method. In the next version of our system, we wish to improve the system computing the indices with the $3 \mathrm{D}$ graphics of facial image and $3 \mathrm{D}$ vector.

\section{Conclusion}

This paper deals with a new assessment method of facial palsy. Man calculated the motion vectors of reference points on the face in the facial expressions, and applied to a quantitative assessment of facial palsy. We used color information for deciding facial position in the image. More precise contour extraction was performed using region extraction method within the facial position with uniform HVC color space. We used characteristic of the facial muscles to decide the reference points on the obtained facial region. We tried to express the precise movement of the reference points along the facial muscles, using region segmentation. We proposed the indices based on the symmetry of the facial motion. Five facial expressions were used for study; Rest, Grin, Whistle, Eye closure and Wrinkling up forehead. Moreover, we performed total assessment by adding synkinesis to indices.

We compared our proposed indices of 5 facial expressions with those of the 40 point method. When we integrated each index as total assessment, we found the correlation of our method with the 40 point method at an extent. We achieved more comprehensive assessment for facial palsy, since we could use more facial expressions than the previous method using contour extraction of facial parts such as mouth and lips. It seems from the obtained results that our proposed motion vector method can be applied to the quantitative assessment of facial palsy, and that it is effective as a diagnosis support system.

\section{Acknowledgment}

This work was supported in part by Grant-in-Aid for Scientific Research (C) No.14571633 by Japan Society for the Promotion of Science (JSPS)

(Manuscript received Dec. 24, 2004, revised Aug. 26, 2005)

\section{References}

(1) J.W. House and D.E. Bradkmann: "Facial Nerve Grading System", Otolaryngol Head Neck Surg, Vol.93, pp.146-147 (1985)

(2) Y. Cui, M. Wan, and J. Li: "A new quantitative assessment method of facial paralysis based on motion assessment", Proc. Annu Int Conf IEEE Eng Med Biol Soc, Vol.20, No.3, pp.1412-1415 
(3) H. Minamitani, A. IIjima, and T. Kunihiro: "Diagnosis System for the Quantitative Assessment of Facial Nerve Palsy Based on the Image Analysis of Facial Movement", Trans. Japanese Society for Medical and Biomedical Engineering, Vol.41, No.2, pp.1-10 (2003) (in Japanese)

(4) T. Tanaka, J. Nemoto, and T. Kunihiro: "Study on Objective Evaluation Method of Facial Nerve Paralysis by Contour Extraction", World Congress on Medical Physics and Biomedical Engineering, WE.TR2-7 (2003)

( 5 ) J. Nemoto, T. Tanaka, and T. Kunihiro: "An Objective Evaluation Method of Facial Palsy by Feature Extraction", IFMBE Proc, Vol.3, No.2, pp.1142-1143 (2002)

(6) M. Isono, K. Murata, M. Kawamoto, H. Miyashita, K. Saito, and K. Yoshikawa: "Objective Evaluation of the Movements of the Muscles Responsible for Facial Expression", Facial Nerve Research, Vol.20, pp.17-19 (2000) (in Japanese)

( 7 ) K. Yuen, I. Inokuchi, M. Maeta, H. Akagi, and K. Nishizaki: "Evaluation of Facial Palsy by the Moiré Topography Index", Facial Nerve Research, Vol.20, pp.11-13 (2000) (in Japanese)

(8) S.M. Newhall, D. Nickerson, and D.B. Judd: "Final Report of the O.S.A Subcommitte on the Spacing of the Munsell Colors", J. Opt. Soc. Am., Vol.33, pp.385-418 (1943)

(9) D.H. Ballard and C.M. Brown: Computer Vision (1987)

(10) N. Otsu: "A Threshold Selection Method from Gray-Level Histograms", IEEE, pp.62-66 (1979)

(11) M. Miyahara and Y. Yoshida: "Mathematical transform of ( R, G, B) color data to munsell (H, V, C) color data", The Journal of The Institute of Television Engineers of Japan, Vol.43, No.10, pp.1129-1136 (1989) (in Japanese)

(12) K. Waters: "A muscle model animating three-dimensional facial expression", Comput Graph, Vol.21, No.4, pp.17-24 (1987)

(13) H. Ohta, H. Saji, and H. Nakatani: "Muscle-Based Feature Models for Analyzing Facial Expressions", Lect Notes Comput Sci, Vol.135, No.2, pp.711-718 (1997)

(14) M.B. Ahmad, D.Y. Kim, K.S. Roh, and T.S. Choi: "Motion Vector Assessment using Edge Oriented Block Matching Algorithm for Video Sequences", Proc 2000 Int Conf Image Process, Vol.1, pp.860-863 (2000)

(15) P. Gunaratne and Y. Sato: "Estimation of Muscle Attachment Points based on Anthropometric Landmarks for Physical Facial Models", Technical Report of IEICE PRMU, Vol.2000-1, pp.1-6 (2000)

(16) P. Gunaratne and Y. Sato: "Quantification of Facial Asymmetry with Mesh based Approach", Conputer Vision and Image Media, No.130-9, pp.63-70 (2001)

(17) P. Gunaratne, T. Amano, and Y. Sato: "Facial Movement Analysis with Iterative Mesy Adaptation", Technical Report of IEICE PRMU, No.2001-116, pp.21-26 (2001)
Toshiyuki Tanaka (Member) received the B.S. in 1982 from

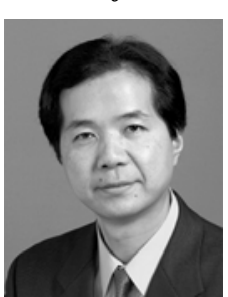
the Department of Instrumentation Engineering, Faculty of Engineering, Keio University. Completed doctoral program in 1989. D.Eng. Instructor 1989, Faculty of Science and Technology. Visiting Researcher 1995-1996 RWTH Aachen, Germany. Now associate professor, Department of Applied Physics and PhysicoInformatics, Faculty of Science and Technology, Keio University. Engaged in research on medical image processing, biometric authentication, signal processing, GPS and its application, and nonlinear oscillation theory.

Junko Nemoto (Non-member) received the B.S. in 2002

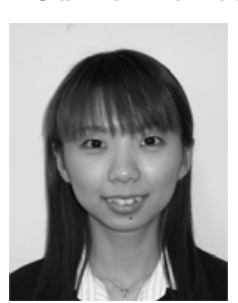
from the Department of Applied Physics and Physico-Inoromatics, Faculty of Science and Technology, Keio University, and received M.S. in 2004 from Shool of Science and Technology, Keio University. Now she works at Canon co. Her research interest at university days is assessment system for facial palsy.

Manami Ohta (Non-member) received the B.S. in 2004

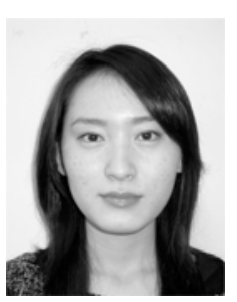
from the Department of Applied Physics and Physico-Inoromatics, Faculty of Science and Technology, Keio University. Now she works at NTT East. Her research interest at university days is assessment system for facial palsy.

Takanobu Kunihiro (Non-member) received the B.S. in 1982 from Faculty of Medicine, Keio University. Trainee doctor 1982 . Instructor 1986. Visiting Researcher 1993-1994 RWTH Aachen, Germany. Now associate professor, Department of Otorhinolaryngology, School of Medicine, Keio University. Engaged in research on facial palsy, dizziness and labyrinthine sense affection. 